\title{
Parabolic Pulse Generation through Passive Reshaping of Gaussian Pulses in a Normally Dispersive Fiber
}

\author{
Christophe FINOT, Lionel PROVOST, Periklis PETROPOULOS \\ and David J. RICHARDSON \\ Optoelectronics Research Centre, University of Southampton, Southampton SO 17 1BJ, United-Kingdom \\ christophe.finot@u-bourgogne.fr
}

\begin{abstract}
We numerically and experimentally demonstrate that a Gaussian pulse can be reshaped into a pulse with a stable parabolic intensity profile during propagation in normally dispersive nonlinear fibers.
\end{abstract}

\section{Introduction}

Pulses with a parabolic intensity profile have recently generated great interest in the field of telecommunications due to their intrinsic resillience to the deleterious effects of optical wave-breaking $[1,2]$, and due to their numerous potential applications in short pulse generation [3, 4], regeneration [5] as well as multi-wavelength pulse generation $[4,6]$. So far various methods have been proposed to generate such pulses both actively by using the asymptotic evolution of any pulse in a normally dispersive fiber amplifier [3, 6], or passively with normally dispersive dispersion decreasing fiber [7], or linear shaping using superstructured fiber Bragg grating [4, 5].

We propose here a new and simple passive method of generating parabolic pulses that relies upon nonlinear propagation in discrete sections of commercially available normally dispersive fibers. We first show how an initial Gaussian pulse evolves during propagation in a normally dispersive fiber and reshapes at a particular point into a pulse with a temporal parabolic intensity profile with a nearly linear chirp. This pulse can then maintain its profile in a second highly nonlinear normally dispersive fiber. Experimental results based on an accurate characterization both in intensity and phase confirm the feasibility of this approach.

\section{Progressive reshaping of a Gaussian pulse in a normally dispersive fiber}

The longitudinal evolution of the complex electric field $u(\tau, \xi)$ of a pulse in a normally dispersive fiber can be modelled by the normalized nonlinear Schrödinger equation (NLSE) [8] :

$$
i \frac{\partial u}{\partial \xi}=\frac{1}{2} \frac{\partial^{2} u}{\partial \tau^{2}}-|u|^{2} u
$$

$u, \tau$ and $\xi$ are normalized parameters defined by :

$$
u(\xi, \tau)=\frac{N}{\sqrt{P_{C}}} \psi, \quad \tau=\frac{T}{T_{0}}, \quad \xi=\frac{z}{L_{D}}, \quad \text { with } \quad L_{D}=\frac{T_{0}^{2}}{\beta_{2}}, \quad L_{N L}=\frac{1}{\gamma P_{C}}, \quad N=\sqrt{\frac{L_{D}}{L_{N L}}} .
$$

$T_{0}$ and $P_{C}$ are respectively the characteristic temporal width and the peak power of the initial Gaussian pulse $\psi(0, T)=\sqrt{P_{C}} \exp \left(-T^{2} / 2 T_{0}^{2}\right) . T, z$ and $\psi$ are the time in a copropagating time-frame, the propagation distance and non-normalized electrical field respectively. The fiber parameters $\left(\beta_{2}\right.$ the second order dispersion and $\gamma$ the nonlinear coefficient) are taken into account via $L_{D}, L_{N L}$ and $N$ which are the dispersion length, the non-linear length and the "soliton" number respectively.

In Fig. 1 we present the evolution of an initial Gaussian pulse with $N=4$. The interplay between dispersion and nonlinearity leads to a progressive reshaping of the pulse. At $\xi=0.2$ (Fig. 1(a3)), the pulse exhibits an intensity profile which is close to a parabolic form, as confirmed by the good agreement between the intensity profile and a parabolic fit which shows only a small discrepancy is the wings of the pulse. Beyond this distance, the intensity profile begins to deviate more strongly from the parabolic shape with a flattening of the top of the pulse (Fig. 1(a4)). 


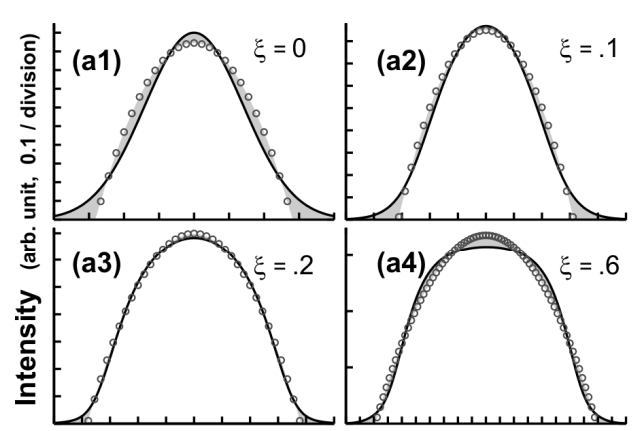

Time $\tau=T / T_{0}(0.5 /$ division $)$

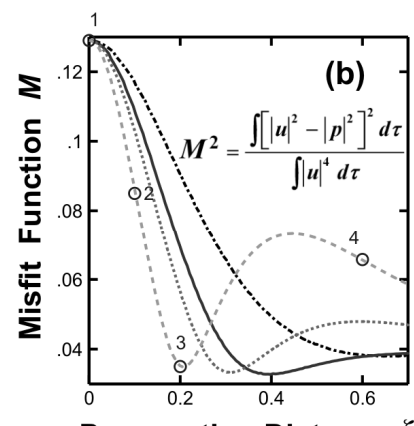

Propagation Distance $\xi$

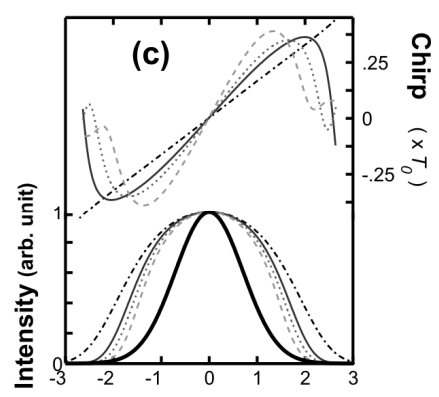

Time $\tau$

Fig 1. (a) Evolution of the temporal intensity profile of an initial Gaussian pulse in a normally dispersive fiber for $N=4$ and for different propagation distances $\xi: 0,0.1,0.2$ and 0.6 (Fig. a1, a2, a3 and a4 respectively). Results from the numerical integration of Eq. (1) (solid lines) are compared to parabolic fits (circles). (b) Evolution of the misfit parameter $M$ versus the normalized propagation distance $\xi$ for different values of $N(N=2,2.6,3$ and 4, mixed, solid, grey dotted and grey dashed lines respectively). (c) Temporal intensity and chirp profiles at $\xi_{\text {opt }}$ for different $N$ values $(N=2,2.6,3$ and 4, same conventions as Fig. 1(b) ). The initial Gaussian pulse is presented in bold solid line

In order to quantify the evolution towards a parabolic shape, we have computed the evolution of the normalized misfit parameter $M$ between the pulse intensity profile $|u(\tau)|^{2}$ and a parabolic fit $|p(\tau)|^{2}$ of the same energy, with $p(\tau)$ corresponding to the normalized intensity profile $|p(\tau)|^{2}=1-\left(\tau / \tau_{P}\right)^{2}$ if $|\tau| \leq \tau_{P}$ and $p(\tau)=0$ otherwise (Fig. 1(b)). We can then see that for any $N, M$ reaches a minimum $M_{\text {opt }}$ after a distance $\xi_{\text {opt }}$ where the pulse has an intensity profile close to the desired parabolic shape. $\xi_{\text {opt }}$ depends strongly on $N$, and so does the behaviour of the pulse after $\xi_{\text {opt }}$. The lowest $M_{\text {opt }}$ is obtained for $N=2.6$.

In order to understand the influence of $N$ on the pulse shape generated at $\xi_{\text {opt }}$, we have plotted in Fig. 1(c), the temporal intensity and chirp profiles for different $N$ values. We note strong differences in the chirp profiles of the parabolic pulses: for low $N$ values, the chirp is linear over the majority of the pulse whereas for higher $N$ some large deviations from a linear behaviour appear in the central part of the pulse. From this non-monotonic chirp profile we can anticipate that the pulse will be subject to wave-breaking deformations [1]. In other words, the proposed method cannot generate a stable parabolic pulse with a high $N$ using just a single segment of fiber.

To overcome this limitation, we propose to first generate a parabolic pulse in a section of fiber under optimal conditions $\left(N_{\text {opt }}=2.6\right.$, which leads to a close to linear chirp across the entire pulse, see Fig. 1(c), solid line) and then to stabilise the parabolic pulse shape using one of two possible approaches: either by amplifying this pulse before further propagation in another fibre, or using propagation in a second fiber with a higher ratio of $\gamma / \beta_{2}$. Under these conditions the pulse propagation in the second segment will be mainly affected by non-linearity ensuring that the linearly chirped parabolic pulses maintain their shape even in the presence of dispersion [2].

\section{Experimental demonstration}

We have carried out the following experimental verification (Fig 2). Nearly-transformed limited Gaussian pulses are generated from a gain switched DFB laser with optimal compensation of the residual chirp. These Gaussian pulses are then amplified to the desired $N$ value before propagation in 50 meters of dispersion compensating fiber (DCF) whose length corresponds to $\xi_{\text {opt }}$ at $N_{\text {opt }}$. Output pulses from this first segment are then characterized in intensity and phase by a second harmonic generation frequency resolved optical gating device (SHG-FROG) [9].

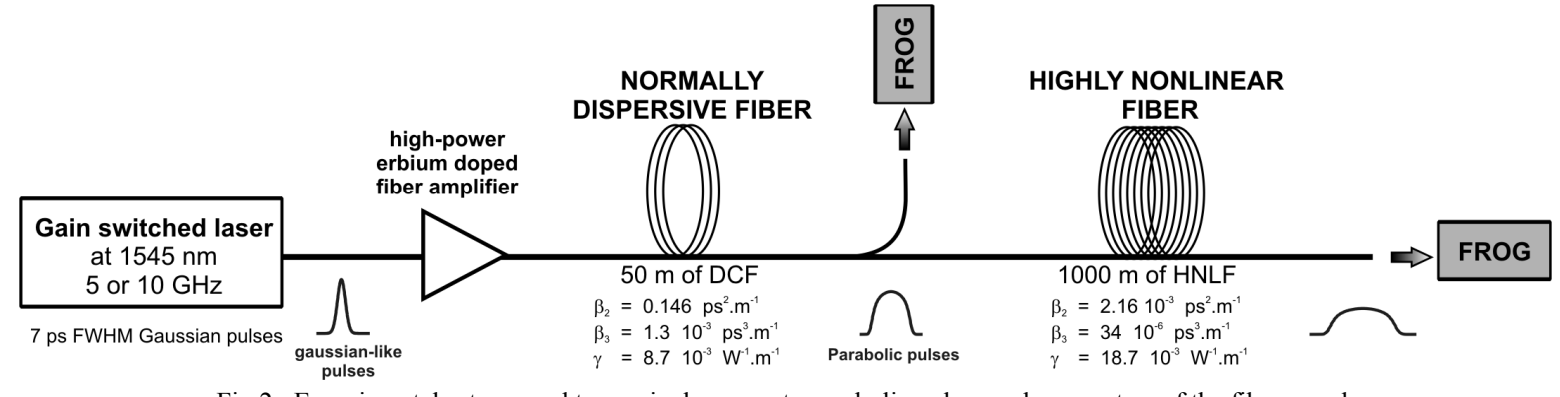

Fig 2 : Experimental set-up used to passively generate parabolic pulses and parameters of the fibers used.

We can see from Fig. 3(a) that a clear reshaping of the pulse into a parabolic form takes place for an initial energy of $47 \mathrm{pJ}$ per pulse $(N=2.6)$. We point out the good agreement with a parabolic fit and with numerical 
simulations of (1) which include also the effects of third order dispersion $\beta_{3}$ and take as an initial condition the input pulse form as experimentally characterized by the FROG technique. For a lower pulse energy $(11 \mathrm{pJ}, N=1.1)$, the pulse remains closer to a Gaussian form whereas for higher energies $(115 \mathrm{pJ}, N=4)$ the top of the pulse becomes flattened.
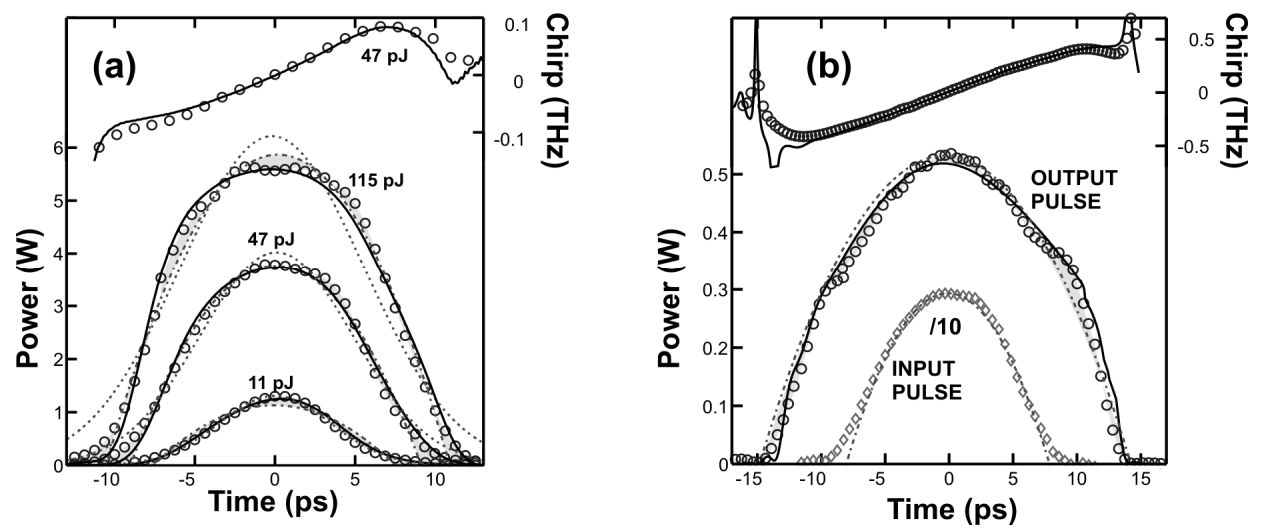

Fig 3 : Temporal chirp (top) and intensity (bottom) profiles. (a) Pulses after the segment of DCF, for initial pulse energy of 11, 47 and 115 pJ. Experimental results obtained by FROG technique (circles) are compared to numerical simulations (solid black line) and to Gaussian or parabolic fits (dashed and mixed lines respectively). $\quad$ (b) Pulses characterized by FROG after the first stage (diamonds) for an initial $\mathrm{N}=2.1$ and after the second stage (circles). Experimental results are compared with parabolic fits (mixed lines) and with numerical simulations (solid black line)

We then investigated the possibility of propagating these pulses in a second section of highly nonlinear fiber without losing their parabolic characteristics. In order to carry out the demonstration at $10 \mathrm{GHz}$, we have used a slightly non-optimal pulse energy $(N=2.1,31 \mathrm{pJ}$ per pulse). The set-up appears however to be tolerant to this and at the output of the first segment, we obtain the expected parabolic profile (see Fig.3b). The FROG characterisation at the output of the second highly-nonlinear fiber confirms that the pulse remains parabolic and has developed a highly linear chirp. These results are in agreement with numerical simulations (third order dispersion, splice and distributed linear losses of the fiber are accounted for in the model).

\section{Conclusion}

We have demonstrated the possibility of generating linearly chirped parabolic pulses in a completely passive manner using progressive reshaping of pulses in commercially available normally dispersive fibers. By carefully choosing the initial power and the length of the fiber, one can expect to have a parabolic pulse. This thus offers a very promising way to generate parabolic pulses at high repetition rates. Our theoretical results are confirmed by experiments which demonstrate the ability of the pulses to maintain their parabolic shape in a second fiber segment in which non-linear effects are predominant. Our approach can be extended to other pulse shapes as long as the initial optimal power and length of the first fiber section are carefully optimized.

\section{Acknowledgement}

Christophe Finot gratefully acknowledges the financial support of European Union through a Marie Curie grant. We acknowledge K. Mukasa for fruitful discussions and Furukawa Electric for the loan of the highly non-linear fiber. This work was funded in part by the European Union Specific Targeted Research Project TRIUMPH IST-027638.

\section{References}

1. W. J. Tomlinson et al., "Optical wave-breaking of pulses in nonlinear optical fibers," Opt. Lett. 10, 457-459 (1985).

2. D. Anderson, M. Desaix, M. Karlson, M. Lisak, and M. L. Quiroga-Teixeiro, "Wave-breaking-free pulses in nonlinear optical fibers," J. Opt. Soc. Amer. B 10, 1185-1190 (1993).

3. M. E. Fermann, V. I. Kruglov, B. C. Thomsen, J. M. Dudley, and J. D. Harvey, "Self-similar propagation and amplification of parabolic pulses in optical fibers," Phys. Rev. Lett. 84, 6010-6013 (2000).

4. F. Parmigiani, C. Finot, K. Mukasa, M. Ibsen, M. A. F. Roelens, P. Petropoulos, and D. J. Richardson, "Ultra-flat SPM-broadened spectra in a highly nonlinear fiber using parabolic pulses formed in a fiber Bragg grating," Opt. Express 14, 7617-7622 (2006).

5. F. Parmigiani, P. Petropoulos, M. Ibsen, and D. J. Richardson, "Pulse retiming based on XPM using parabolic pulses formed in a fiber Bragg grating," IEEE Photon. Technol. Lett. 18, 829-831 (2006).

6. Y. Ozeki, Y. Takushima, K. Aiso, K. Taira, and K. Kikuchi, "Generation of $10 \mathrm{GHz}$ similariton pulse trains from 1,2 km-long erbium-doped fibre amplifier for application to multi-wavelength pulse sources," Electron. Lett. 40, 1103-1104 (2004).

7. T. Hirooka, and M. Nakazawa, "Parabolic pulse generation by use of a dispersion-decreasing fiber with normal group-velocity dispersion," Opt. Lett. 29, 498-500 (2004).

8. G. P. Agrawal, Nonlinear Fiber Optics, Third Edition (San Francisco, CA : Academic Press, 2001).

9. R. Trebino, Frequency-Resolved Optical Gating : the measurement of ultrashort laser pulses (Norwell, MA : Kluwer Academic Publishers, 2000). 